\title{
Fire prevention and protection in civil buildings: the VALERIE project
}

\author{
G. Bochicchio ${ }^{1}$, R. Lenzi ${ }^{2}$, M. Nart ${ }^{1}$, T. Zuccaro ${ }^{3}$ \& A. Ceccotti ${ }^{1}$ \\ ${ }^{1}$ CNR-IVALSA Consiglio Nazionale delle Ricerche, \\ Istituto per la valorizzazione del legno e delle specie arboree, Italy \\ ${ }^{2}$ Corpo permanente dei Vigili del Fuoco della Provincia Autonoma di \\ Trento, Italy \\ ${ }^{3}$ ISAQ Studio, Italy
}

\begin{abstract}
The "VALERIE" project ("Fire Risk Assessment - Fire reaction of construction products") originates from a strict co-operation between IVALSA, the Fire Brigade Department of Trento and ISAQ Studio in Falconara (Ancona - Italy). The main objective of this project is to implement a standard procedure to reduce fire risks in civil buildings and to do this by applying the most efficient technology or the most cost efficient methods, taking into account the limitation of the regulation. The methodology proposed is quite user-friendly, even if it requires a good deal of experience about the fire risk analysis. Qualified professionals are therefore required for an adequate application of the VALERIE approach, consisting of a multi-level hierarchical analysis whose application implies the assignment of the appropriate "weight" to each element into which the whole problem (i.e. the fire safety of that specific building) has to be decomposed. The quality of the results given by the VALERIE model is directly related to appropriate choice of these "weights". To guarantee an unbiased assessment of the influence of each parameter relevant to the fire safety, a "reference ranking" shall be built-up, which has to be dependent from actual local working conditions. VALERIE, in order to obtain a common working platform, aims for a strict cooperation both with the fire brigade and with professionals in charge of the approval of the submitted designs. A further important task of this project is the definition of reference parameters for the assessment of a "safety index" recognised by all the involved parts and included in the official approval documents.
\end{abstract}

Keywords: fire risk assessment, analytical hierarchical process, fire safety engineering, efficiency index. 


\section{Introduction}

The VALERIE project was developed with the purpose of identifying an operational tool to make it possible to assess the level of safety guaranteed by fire prevention design or by an existing construction, namely conditions inherent in the construction system relating to the safety of users, together with protection and prevention of damage resulting from accidental fires. The method aims to be as logical and objective as possible in terms of evaluating anomalous or unregulated cases, in the case of exceptions to technical rules or as regards compromises, at times necessary when the building in question is part of the cultural heritage. However, it was also intended as an operating system for quantitative evaluation of the influence of design choices on fire safety, thus abandoning the qualitative experience-linked approach still adopted. It is the result of close cooperation between several working groups funded by the Autonomous Province of Trento and coordinated by IVALSA (Istituto per la valorizzatione del legno e delle specie arboree), an institute in the network of the Consiglio Nazionale delle Ricerche (CNR).

The project is in line with the engineering approach to fire prevention and safety, recommended for some time by the European Union in the "Interpretative document for essential requirement no. 2 - safety in case of fire". It makes general reference to the DM of 14/01/2008 "Technical regulations for constructions": $\S 3.6$, entitled "Accidental action", indeed allowing for the possibility of adopting a performance-based approach to design according to fire safety engineering criteria (FSE), through a balanced scale of active and passive protection measures". The essential requirement "Safety in case of fire" is considered to be satisfied in the case that the construction complies with regulations referring to this requirement, transferring the requirement into limitations. In the event there are no applicable regulations, evaluation is carried out by the designer. All this naturally assumes that there is an appropriate level of reliability in terms of execution; it surmises that suitable measures guaranteeing quality are considered, in order to provide a system corresponding with the requisites and the ideas formulated during the design process.

The objective of the project is the creation of a decision-making model designed to optimise resources, with the main scope of reducing the risk of fire. Fire prevention is currently designed in a simplistic manner, above all when there is no technical regulation: indeed the measures suggested by the designer derive from straightforward technical evaluation (technical judgement) based on experience and good sense, isolated from any quantitative controls. The same may be said of the controlling activities of the fire service, so evaluation is strongly subjective and not particularly objective. It is clear that technical judgement will always be irreplaceable, given that it is not possible to reduce design to a mechanically executable algorithm; indeed the tendency in Europe is to grant increasing space to the freelance professional, in terms of margins of discretion and consequential responsibility, limiting public checks to assessment of the general aspects most significant in terms of safety, without going into detail and specific design choices. However this does not exclude the possibility 
of quantitative control of the decision-making process, indeed the first is a valid support for the second. Fire prevention measures are frequently analysed individually, without correctly considering the context. Nor, on the other hand, do technical regulations specify quantitative measurement of the level of safety that the legislator considers acceptable. The decision-making model analysed provides for a link between objectives, strategies and measures, allowing evaluation of the efficiency of fire prevention design, with the possibility of also including financial parameters in order to obtain genuinely significant responses.

International and European regulatory organisations have dealt with the problem of performance-based evaluation of fire prevention design, leading to the issuing of various technical regulations, including ISO series 13387 and ISO 13943. CEN has also expressed itself, albeit in part, within Eurocode 1 (UNI EN 1991-1-2:2004) "Action on structures - Parts 1-2: Action in general - Action on structures exposed to fire", which introduces the concepts of fire scenarios and fire design (subsequently picked up on in the DM of 14/01/2008), to be determined on the basis of risk assessment and for the purpose of which advanced fire models can be used.

\section{Methodology of the VALERIE project}

The method proposed has been developed on the basis of Analytic Hierarchy Process (AHP) method.

The Analytical Hierarchical Process is a practical technique for modelling and solving multi-criteria decision problems. The AHP approach facilitates the development of logical hierarchical structures for complex decisions. A logical framework is set up, which allows improving the performance of complex decisions by decomposing the problem in a particular hierarchical structure. The incorporation of all the decision criteria, allows the decision maker to determine trade-offs among objectives.

An analysis of this type is concretized in the construction of a matrix, whose lines bring the different alternatives while in the columns there are the criteria of judgment defined with regard to the different finalities previously fixed. The generic element of the matrix gives the measure of the choice considered with regard to that criterion, which is the measure of the effect that this offers as for objective.

The problem is decomposed in a particular hierarchical structure organized on different levels that consist of different elements to each of which is assigned a weight with regard to the element that belongs to the level above. In this way, both the principal goal and precise solutions that concern partial aspects are obtained.

A decision method that follows an approach of this type facilitates the development of hierarchical logical structures in the case of complex decisions, quantifies the efficiency of evaluations and opinions to sift, assigns weights, and therefore influences, to different possibility in comparison to others.

Each process that for its nature is systematic can be analyzed using the AHP. The hierarchical structure is a representation of the formal properties and the 
relationships among the components to facilitate the analysis of their impact as regards of complete system. Such abstraction has the form of a pyramid, to whose apex always a principal goal, but whose structure is unique for every system analyzed in terms of objectives, strategies and measures.

The attribution of the relative importance, that is weights, of each parameter at each level, depends from the type of problem that we want to solve.

The problem of evaluating fire safety is simplified using a specific hierarchical structure organised on four levels: the main goal it is intended to achieve, namely fire safety; objectives directly linked to the main goal, namely the action necessary for the success of the established goal; the strategies making it possible to implement the objectives and finally fire prevention measures, namely the practical solutions necessary to implement strategies.

In particular, the efficiency of fire prevention measures is evaluated using the degree of satisfaction associated with them. The degree of satisfaction $G$ indicates to what extent the measure linked to it is effectively present in the design. It will be 0 if the measure is actually completely absent and 1 if fully satisfactory, taking on intermediate values in other situations. The definition of $\mathrm{G}$ follows precise rules dictated by technical regulations, the technical criteria for fire prevention and FSE, therefore representing a parameter which is as objective as possible. For the purposes of verifying the design it must therefore be equal or greater than a minimum level defined on the basis of vertical regulations to which the analysed activity is subjected.

Each element in the structure is assigned with a weighting depending on the element belonging to the higher level. The allocation of a numerical value indicates the importance or influence that each parameter has as compared to the parameter on the higher level. The method supplies reference tables; however, the values considered are only indicative as the definitive value can only depend on the real case it is intended to assess.

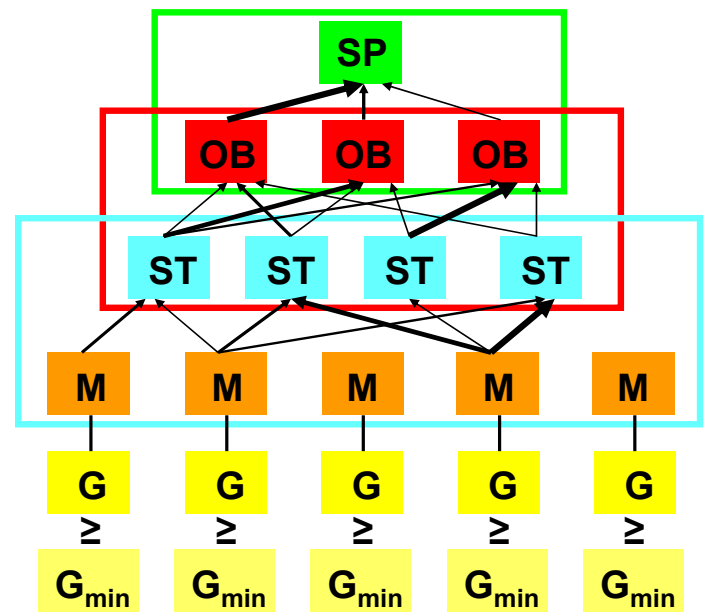

Figure 1: Structure of the VALERIE method: input. 
The general question it is necessary to answer in order to choose the value for each objective, strategy or measure as compared to the main goal, the objectives or the strategy is:

"How much does this element influence the possibility of achieving the established goal, objective or strategy?"

The answer obviously depends on political and socioeconomic choices, on the environment in which the building is situated but also on the characteristics of the design or the building itself.

To facilitate the process of assigning values, reference has been made to the scale of values already proposed by Prof T. Saaty, who established a classification from 0 to 9 with the increasing influence of the element, thus transforming qualitative evaluation into numerical values.

These are then standardised in order to obtain values between 0 and 1 , the final sum of which must clearly be equal to 1 . In this way there is interaction between the parameters on the same level as compared to those on the higher level. It is thus possible to evaluate the impact of each level on the preceding one, and to define priorities within the same level as compared to the main goal established.

The input is the weight that every measure has on the strategies, the weight that every strategy has on the measures and also the weight that every objective has on the principal goal.

The output that draws corresponds to the weight that every strategy has on the principal goal and to the weight that every measure has both on the principal goal and on the objectives

In particular, an initial result will be a standardised scale for the weighting of elements on the level of fire prevention measures as compared to elements on level 2, objectives. This gives a scale of priorities identifying the most vulnerable measure in the design, the one most involved, but also classification of all those considered in relation to their efficiency.

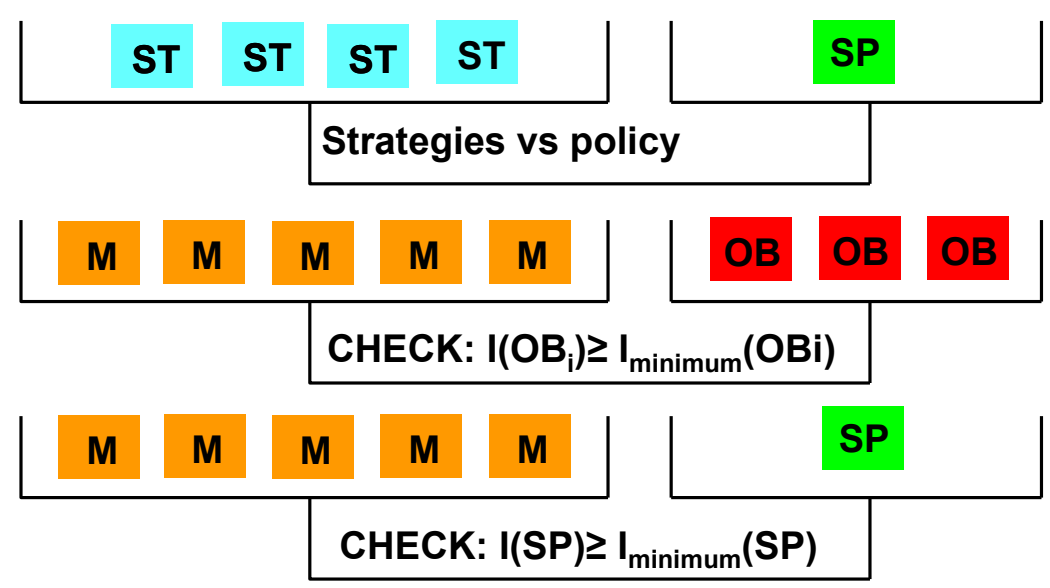

Figure 2: $\quad$ Output. 


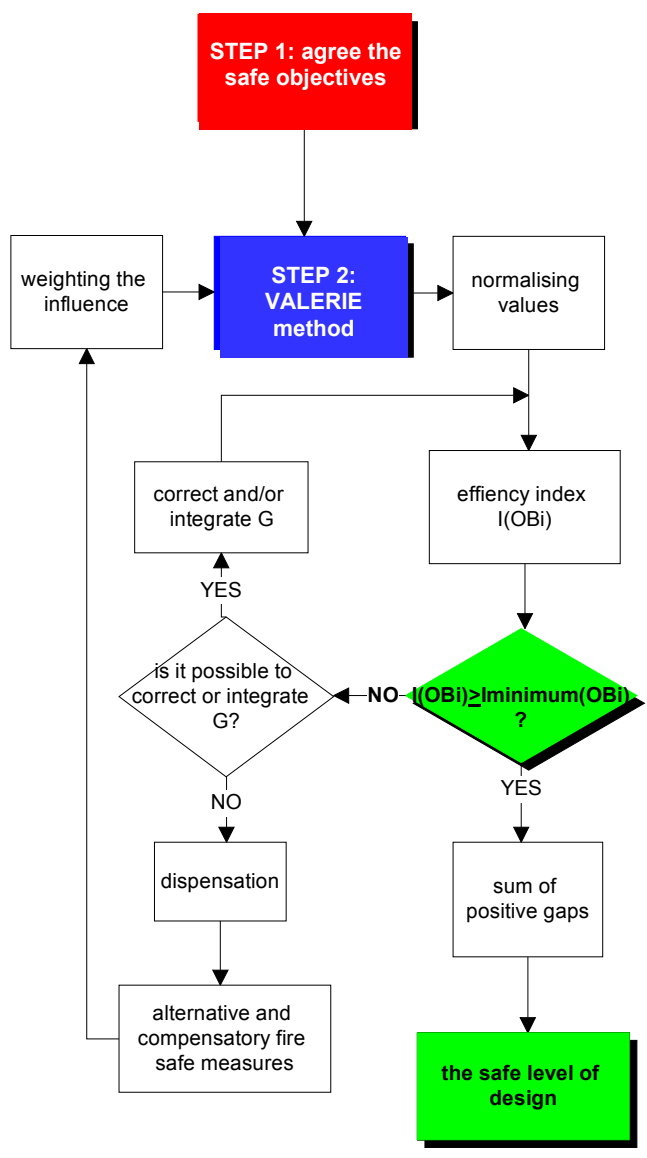

Figure 3: VALERIE procedure.

What is more, respecting the technical regulations, each objective must be reached, establishing a mandatory threshold; if an objective is reached and exceeded, this advantage cannot compensate for the failure of another objective to reach its target.

Thus the final result of the applied method will be the achievement of the main goal, fire safety, evaluated in terms of the efficiency of the design and given by the sum of the deviation, which may not in any case be negative, between the level reached and the minimum threshold required for each objective.

If the minimum threshold imposed by the regulations for one of the objectives is not reached, the project, as contemplated by the same regulations, must ask a dispensation. 
Therefore when is not possible to correct and/or to integrate the fire safety measures to change the values attributed to the grade of satisfaction, the authority can appraise the possibility to foresee alternative and compensatory measures.

In line with what has been stated, the result obtained must necessarily consider the models of calculation presented in fire safety engineering (FSE), statistical evaluation and the reliability of the fire prevention measures present on the market, the results of certain experimental tests, the models adopted for risk assessment and the provisions of the relevant regulations.

VALERIE represents a tool for checking design decisions, thus avoiding as far as possible subjective evaluation unsupported by concrete elements, to instead consider them within a carefully considered and relational context.

The hierarchical structure proposed is thus defined on the basis of the procedural aspects and the criteria to be adopted to evaluate the level of risk and the design of the consequential compensatory measures, as reported in the DM already cited.

\subsection{Hierarchy network}

The structure is given below:

$1^{\text {st }}$ level: main goal

$2^{\text {nd }}$ level: objectives

\section{PO1 fire safety}

$O B 1$ to protect people, guaranteeing their safety throughout the time they are expected to remain in the building

$O B 2$ to protect fire service staff, guaranteeing the safety of emergency and fire service teams in every phase of intervention

$O B 3$ to protect the building, if possible avoiding its collapse in the event that it is of historic, artistic or architectural merit

$O B 4$ to protect the content, if this should be of historic or artistic value

$O B 5$ to safeguard the continuation of activities to allow possible reuse of the structure when required

$O B 6$ to safeguard the surrounding environment, hence air, water, $3^{\text {rd }}$ level: strategies surrounding buildings and the urban environment

ST1 to reduce the likelihood of a fire starting to a minimum, including all measures necessary to minimise the risk of possible fires being sparked off

ST2 to limit the spreading of fire, including all measures to delay the development of the fire or limit it to the compartment where it began, but also to prevent the fire from spreading to other compartments on the same floor, to other floors or other buildings

ST3 to facilitate escape routes, including all measures contributing towards making faster and safer the movement of people present in the building towards a place considered secure 
ST4 to facilitate extinguishing of the fire and rescue operations, including all measures which may facilitate both emergency intervention by staff responsible for fire-fighting present in the building and intervention by the fire service

ST5 to limit the effects of products of the fire, including all measures necessary to limit the effects of smoke on people, buildings and content, together with possible contamination of water and the environment

ST6 to facilitate the speed with which a fire is located, including all measures which make it possible to swiftly identify the exact spot where the fire started and the cause of the fire

ST7 to protect fire prevention components and systems, including all measures guaranteeing that they also function $4^{\text {th }}$ level: measures during a fire

M1 reaction of internal and external cladding materials and furnishings to fire, indicating their degree of participation in the fire

$M 2$ resistance of structures to fire

M3 resistance of partitions to fire: in relation to the resistance of all elements expected to have a certain ability to limit the spreading of the fire to other compartments. Hence the ability of the element to remain intact and retain its thermal insulation, together with other possible criteria, also with the scope of protecting escape routes

M4 size of compartments

M5 characteristics and location of doors and windows on the facade, to evaluate the possibility of fire spreading to the environment surrounding the building or to other floors of the building through these openings. The presence of balconies also influences this

M6 characteristics and location of doors and windows in rooms in the building, indicating the ability of the environment to discharge heat through vertical ventilation (ventilation factor)

$M 7$ distance between buildings and separating structures, in relation to the possibility of the fire spreading to other buildings, a crucial factor above all in urban areas and historic centres M8 geometry of escape routes, this regards the ease with which rapid escape is possible from the building, hence the width of corridors and stairs, the distance between any point in the building and the safety exits, the number and distribution of safety exits

M9 access for the fire service, to facilitate extinguishing procedures, this regards the width and organisation of access routes, the points where it is possible to enter the building, the 
possibility of approach with a revolving ladder and the presence of any obstacles to bear in mind

M10 detection and warning systems, this regards the speed with which occupants are alerted and the fire service is informed. This category may also include intrusion detection systems in order to protect against arson

M11 extinguishing systems. This takes into consideration automatic extinguishing systems, hydrant networks and mobile extinguishing devices (fire extinguishers)

M12 natural and mechanical control of smoke and heat, maintaining a clear view during evacuation and assistance procedures, making it possible to reduce the amount of smoke and combustion gas present in the environment and to confine them at a height from the floor which allows freedom of movement. This also guarantees protection from the thermal effects of hot smoke and gases for structural elements and assets, thus reducing the risk of structural collapse

M13 alarm systems

M14 in-company fire protection teams

M15 proximity and type of public fire-fighting facilities

M16 maintenance of safety systems

M17 training of staff and occupants, so that they behave correctly both as regards fire prevention and during evacuation procedures

M18 emergency plans for evacuation, in order to establish the correct procedure in every situation

M19 administration of rescue procedures, operations designed to limit the damage to contents and testing of the procedures to be followed in the event of fire in order to save material of historic and artistic value

M20 periodic inspections of the building, to identify all possible sources which might spark off a fire

M21 safety lighting, considering both the position of sources of safety lighting and the level of lighting, but also the visibility of signs and the autonomy of the source

Measures M14, M16, M17, M18, M19, M20 relate to the safety management process within the construction, namely to the overall structure of operational phases following one another, starting from the time such a construction comes into use, with the scope of ensuring that it functions with an adequate level of safety until the end of its practical and economic life-cycle.

The hierarchy contains the same objectives given in the DM of 09/03/2007 "Performance in terms of the fire resistance of constructions in activities subject to control by the national fire service", although in greater detail. For example, in the DM the objectives include "the stability of load-bearing elements for a period of time useful for ensuring aid to occupants", which in the method structure is considered a strategy if related to aid to occupants and an objective if referred to 
safeguarding of the construction. This difference, purely formal, is necessary simply in order to guarantee greater precision in the definition of all aspects relating to safety in the fire protection design.

\section{The safety of the project and the risk analysis}

All factors influencing the level of risk in the situation analysed are indeed implicit in the objectives set out in VALERIE: the use of the building, it's historic and artistic value and its content, the characteristics of the surrounding area, the size of the building, the possible number of people using it and the safety measures provided for within it.

The method thus has the same objectives characterising risk assessment, namely to identify all those elements which may directly or indirectly determine the vulnerability of the design and a potential loss of safety.

It is possible to talk specifically about interdependence of factors, their influence or weight, the practicality of the measures implemented through assessment of their efficiency and suitability with definition of the degree of satisfaction, evaluation of possible alternatives and above all respect for fire prevention regulations.

Some criteria and parameters values descend from experience in fire extinguishment, as well as from specialistic technical judgment in order to suitableness of installations and functional performance, in close connection with general principles of fire safety.

In this situation it is possible to surmise a safety criterion making it possible to recognise whether the design can effectively be considered to have been evaluated or not.

The method gives rise to an index of efficiency for the design, measuring its degree of safety and assigning it with a certain percentage, which is moreover fully comparable with the residual acceptable risk, calculated for each objective and directly for the main goal, using the formulas given below:

$$
\begin{aligned}
& I_{\text {efficiency }_{(O B i)}}=O B_{(i)} \sum_{j=1}^{7} \sum_{k=1}^{21} S T_{(j i)} \cdot M_{(k j)} \cdot G_{(k)} \\
& I_{\text {efficiency }_{(S P)}}=\sum_{i=1}^{6} \sum_{j=1}^{7} \sum_{k=1}^{21} O B_{(i)} \cdot S T_{(j i)} \cdot M_{(k j)} \cdot G_{(k)}
\end{aligned}
$$

where $G(k)$ is the degree of satisfaction relating to each measure $M(k), M$ the fire prevention measures, ST the strategies and OB the objectives.

\section{Conclusion}

The main goal of the VALERIE project is to reduce the fire risks. The method uses to some extent existing evaluation techniques and models which are analysed and adapted. The final result is a procedure that allow for a large degree of flexibility. 
The input of information in the VALERIE method can range from straightforward expert opinion to a more thorough fire safety engineered approach. It is clear that the reliability of the output is governed by the quality of the information input. However, the experience gained during the case studies showed that even a rough approach can provide useful results.

Moreover the evaluation of an effective index has a number of consequences. It is indeed possible to:

- check the safety of the design, comparing the result with the threshold values established for each objective

- immediately identify the fire prevention measure which is most influential for the objective and as regards which it is necessary to act

- justify design choices that offer better safety as compared to the minimum requirements imposed by the regulations, with costbenefit assessment, establishing a plan of priorities to be followed in the event that a fixed budget is applicable.

In particular, cost-benefit evaluation, according to the scheme given below, makes it possible to optimise the resources available, calculating the effective benefit of the individual measure as compared to the cost of its implementation, or of all the measures implemented as compared to total costs, a consideration that it is possible to define both in relation to a single objective and as regards the main goal.

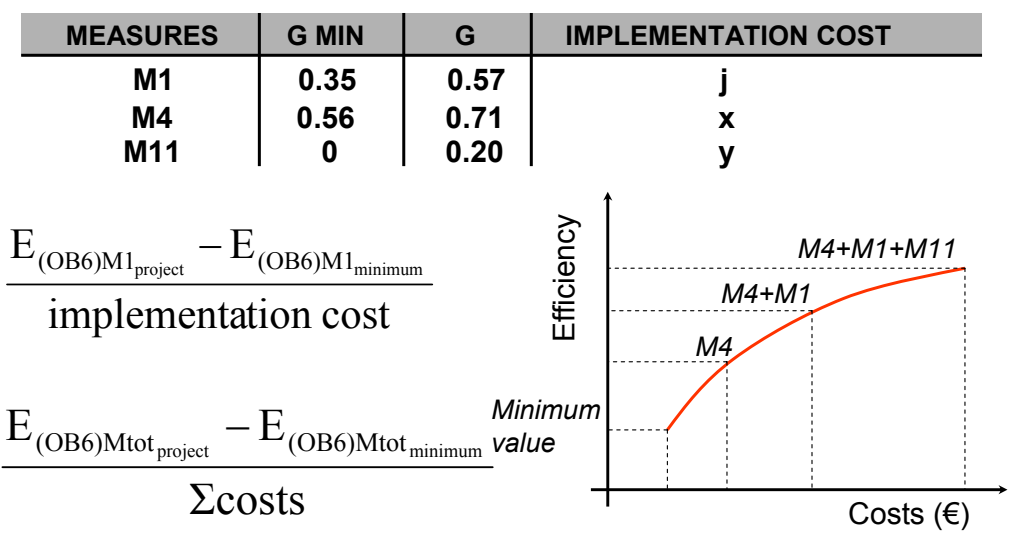

Figure 4: Cost-benefit evaluation.

The measures implemented in relation to threshold values may also be put in order, starting with the measure which has most influence on the safety of the design in relation to its cost and following with the others. In this way it is possible to have a picture of the priorities which respects the budget available, as already underlined.

This ranking may lead to interesting new insights: measures that only give a small improvement of the overall fire safety may be attractive because the costs 
are low. If several of these small measures may be taken, the fire safety level can be greatly improved with limited costs. On the other hand, a single measure that is deemed to greatly improve the overall fire safety may not be attractive if the costs are very high. The highest possible fire safety level will be achieved by selecting as many as possible of the top-ranked measures, up to the available budget.

What is more, the possibility of determining the index of efficiency as indicated above makes it possible to implement the passage from a concept of efficacy and hence of ability to produce the effect and results desired, to one of efficiency, with optimisation of the relationship between the results obtained and the resources employed.

\section{References}

[1] Shields J and Silcock G., "An Application of the Hierarchical Approach to Fire Safety”, Fire Safety Journal, No. 11:33, pp. 235-242, 1986

[2] Dodd F.J. and Donegan H.A., "Prioritisation Methodologies in Fire Safety Evaluation", Fire Technology, Vol. 30, No. 2 / May, pp. 232-249, 1994.

[3] Zhao C.M., Lo S.M., Lu J.A. and Fang Z., "A simulation approach for ranking of fire safety attributes of existing buildings", Fire Safety Journal No. 39, pp. 557-579, 2004

[4] Saaty T. L., The Analytic Hierarchy Process, McGraw Hill, New York, 1980 . 